\title{
Do people use reverse psychology? An exploration of strategic self-anticonformity
}

\author{
Geoff MacDonald, \\ University of Toronto, Canada
}

Paul R. Nail

University of Central Arkansas, Conway, AR, USA

\section{Jesse R. Harper}

University of Western Ontario, Canada

This research examined reports of the real-world use of reverse psychology, or what we term strategic self-anticonformity (SSA). In Study 1, participants reported examples in which they engaged in SSA and rated the success and frequency of use of this influence tactic. These data suggested the existence of two forms of SSA, one used as a general persuasion tactic and one used specifically to garner interpersonal reassurance. Study 2 compared the prevalence of these two forms of SSA with the use of three previously established influence tactics (i.e., door-in-the-face, foot-in-the-door, and disrupt-then-reframe). The results suggest that SSA is a prevalent, realworld influence tactic deserving of further study.

Keywords: Persuasion; Compliance; Anticonformity; Reverse psychology.

One important tradition in the study of social influence tactics is the compliance paradigm (e.g., Davis \& Knowles, 1999; Freedman \& Fraser, 1966). Here the focus is on direct requests from one individual to another, with the requester being of equal or lower status than the receiver (Nail, MacDonald, \& Levy, 2000). Effective influence techniques that have been discovered employing the compliance paradigm include foot-in-the-door

Address correspondence to: Geoff MacDonald, Department of Psychology, University of Toronto, Toronto, Ontario, Canada M5S 3G3. E-mail: gmacdonald@psych.utoronto.ca

This work was supported by a Social Sciences and Humanities Research Council of Canada Standard Research Grant to the first author. G.M. is grateful to the Canadian government for its funding of social science research. Thanks go to Kelly Lawrence and Jess Templeman for their assistance in coding.

(C) 2010 Psychology Press, an imprint of the Taylor \& Francis Group, an Informa business http://www.psypress.com/socinf

DOI: $10.1080 / 15534510.2010 .517282$ 
(FITD; e.g., Freedman \& Fraser, 1966), in which compliance with a small request increases compliance with a later, larger request; door-in-the-face (DITF; e.g., Cialdini et al., 1975), in which noncompliance with a relatively large first request increases compliance with an immediate, smaller request; and disrupt-then-reframe (DTR; e.g., Davis \& Knowles, 1999), in which a request is phrased in unconventional terms then reframed to the advantage of the influence source.

The present research is concerned with a compliance technique that is new to the literature to our knowledge - what we call strategic self-anticonformity (SSA). Situations calling for the potential use of SSA can occur, we hypothesize, when an influence source knows to expect contradiction, negativity, or disagreeableness from an influence target. One such case is highlighted by Brehm's (1966) reactance theory. This theory suggests that an individual who feels a threat to his or her sense of freedom will experience discomfort that motivates re-establishment of that freedom. Such reactance motivation should increase the likelihood of nonconformity to influence attempts. In these situations an influence source may have success by misrepresenting his or her true desires assuming that the target's proclivity for disagreement will result in the target adopting the position that the source secretly desires. For example, parents may feign mild displeasure with their rebellious teenager's new romantic interest, all the while secretly hoping that their lack of enthusiasm will in fact promote the relationship.

Although new to the social influence literature, our own casual observations and reports from others indicate that SSA is familiar to many laypeople under the name reverse psychology. One goal of this paper is to seek more systematic evidence bearing on SSA as a genuine, real-world influence tactic. Another is to compare the reported use of SSA with previously established influence tactics: FITD, DITF, and DTR.

One feature that may differentiate SSA from influence tactics better established in the literature is that SSA is a relatively indirect form of influence. To illustrate, consider a circumstance where you and a friend are considering which of two movies to see together and you have a preference for one over the other. If you know your friend to be a generally agreeable person, then a direct influence strategy should have a reasonable chance for success. You should merely suggest your preferred movie, along with sound supporting arguments. Because the receiver is agreeable, he or she should likely comply with you unless your friend genuinely prefers a movie other than your choice and has better counter-arguments than your original arguments. Here we might refer to your strategy as self-conformity. Anticipating cooperation and agreement from your friend, you can present a position that is consistent with, or one that is in self-conformity with respect to, your true initial preference in suggesting which movie to see. 
However, what if you know your friend to be a generally disagreeable person, one who consistently and habitually battles others for dominance and control? Here it would appear that a self-conformity strategy on your part might backfire. If you state your true preference for Movie A, for example, your friend might well take an opposing view and stubbornly argue for Movie B. We submit that one way out of such a predicament is to employ strategic self-anticonformity. For example, if you want to see Movie A, you might first suggest Movie B. Now when your friend takes an opposing position and argues for Movie A, you can put up a spirited but disingenuous defense before eventually appearing to accede to your stubborn friend's choice for Movie A, your preferred movie all along. In short, if a requester anticipates agreement from a receiver, a direct approach in terms of self-conformity appears to be a reasonable opening strategy. In contrast, if a requester anticipates automatic disagreement from a receiver, an indirect approach in terms of self-anticonformity should work better.

The phrase strategic self-anticonformity is adapted from the diamond model of social response (Nail \& Van Leeuwen, 1993; Willis, 1965; see Figure 1), and connotes the idea that the initial stated position of the requester is the opposite of his or her true position. Whereas the diamond model describes possible responses to social influence, however, our perspective suggests this model can also represent possible stimuli in anticipation of another's response to influence. Consistent with our analysis of possible interpersonal influence situations above, if a requester anticipates agreement (i.e., conformity) from a receiver, then the requester can just state his or her true position (i.e., independence or self-conformity) in the initial influence communication. However, if a requester legitimately anticipates disagreement (i.e., anticonformity), the requester should be more successful opening with the opposite of his or her true position (i.e., self-anticonformity). Thus, considering possible stimuli (Ss) and responses (Rs), there appear to be two logical and natural links between pairs of responses from the diamond model's pure responses. Anticipating agreement, there is an S-R link between independence (i.e., self-conformity) and conformity for the requester and receiver, respectively. Anticipating disagreement, however, there is an $\mathrm{S}-\mathrm{R}$ link between self-anticonformity and anticonformity, respectively.

Despite the familiarity of the concept of reverse psychology in popular culture, no research has investigated the prevalence or effectiveness of its use in everyday life. However, strategic self-anticonformity is used with some regularity as part of a psychotherapy technique known as "therapeutic paradox" or "paradoxical intention" (Ascher, 1989; Frank1, 1967; Loriedo \& Vella, 1992), suggesting that SSA may indeed be useful as an influence technique. Despite the lack of formal empirical evidence for SSA, we have 


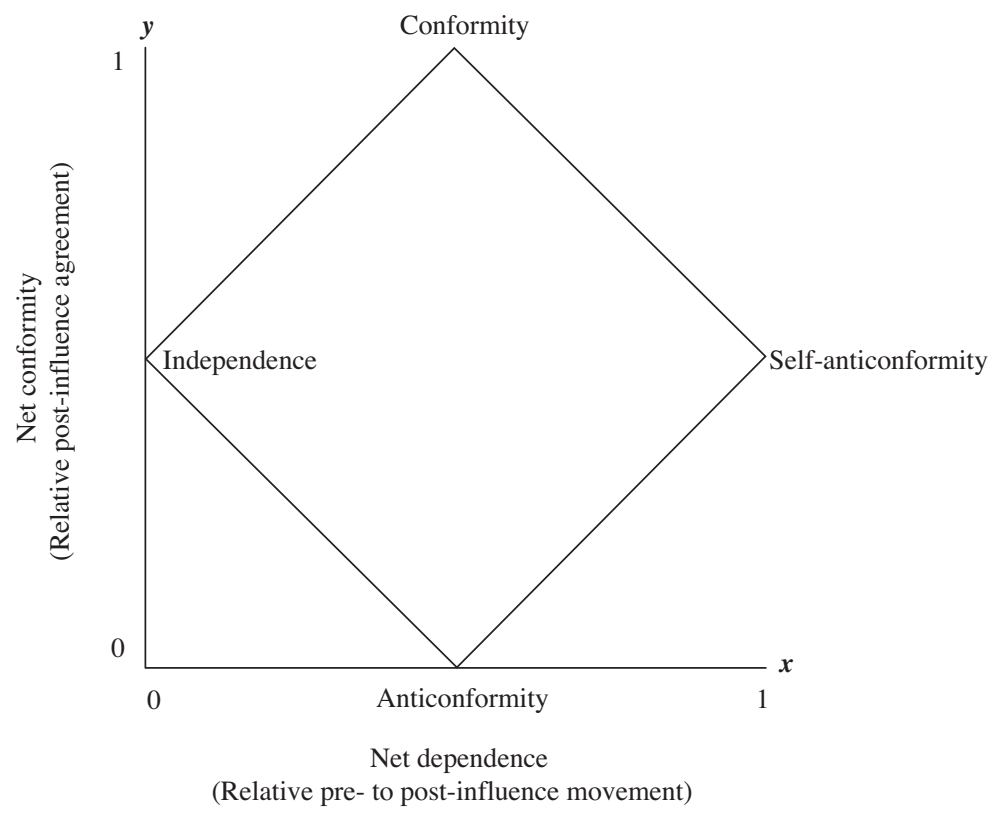

Figure 1. The diamond model of social response. The version shown is the restructured version of the model. From "An Analysis and Restructuring of the Diamond Model of Social Response" by Paul R. Nail and Marilyn Van Leeuwen, 1993, Personality and Social Psychology Bulletin, 19, p. 112. Adapted by permission.

uncovered a number of anecdotal accounts that support its use. For example, in his autobiography country music singer/songwriter Mel Tillis reports that his father was "endlessly contrary" toward his mother, always disagreeing with what she wanted, apparently out of spite (Tillis \& Wager, 1984, p. 185). If Tillis's mother wanted to go out to eat barbecue, for instance, his father would take her to a Mexican restaurant, and vice versa. Eventually, Tillis reports, his mother learned to say the opposite of her true choice (i.e., to self-anticonform); his father would then characteristically disagree (i.e., anticonform), and his mother would end up getting to eat where she had initially desired.

But how pervasive is SSA as an influence tactic? What percentage of people in a sample might report actually having used such a strategy? We attempted to answer these questions in two studies. In Study 1 we described SSA to participants and asked them to provide us examples of their own use of this tactic. In Study 2 we refined our description of SSA based on Study 1's results, and compared individuals' reports of SSA use to their reported use of the more established tactics of FITD, DITF, and DTR. 


\section{STUDY 1}

\section{Method}

\section{Participants}

Participants were 159 undergraduate students recruited from the University of Queensland participant pool who received course credit for their involvement in the study.

\section{Procedure}

Participants were invited to participate in a study on their interactions with others. The study consisted of one questionnaire booklet. On the first page of the booklet, participants were asked to recall an example of their own use of SSA. Specifically, the instructions read as follows:

We're interested in the ways that people interact with each other. One way of looking at the social world is to think of it as each person frequently trying to get others to agree with the way he or she sees the world. Politicians and advertisers do this all the time, but so do individuals. We'd like our friends to agree to see the movie we want to see, to go to the restaurant we'd like to go to, or to share our opinions on things that are important to us. What we're specifically interested in for this study is whether people sometimes say the opposite of what they believe as a way to get others to agree with them. For example, a mother might tell her rebellious daughter that she dislikes her daughter's new boyfriend (who, in reality, she likes), knowing this is likely to increase her daughter's attraction to him. There's also the familiar example of a person saying they look unattractive or overweight in hopes of getting others to disagree and praise their appearance.

What we'd like you to write about below is a description of a time that you tried to get someone to agree with you or to do something you wanted them to do by advocating the opposite, as in the examples above. Please do not limit yourself to an example similar to the ones we described above. We're interested in a wide range of instances, so please take a minute or two to think, and try to come up with a different type of example than we've given. However, if that's the only type of example you come up with, please write about it. If you are unable to think of an example, just leave the space blank. When you have finished writing about your example, please answer the questions on the next page.

After completing this page, participants went on to answer a series of questions about the example they described. 


\section{Measures}

Difficulty. Participants responded to the item, "How difficult was it for you to come up with an example?" $(1=$ extremely difficult, to $9=$ not at all difficult).

Success. Participants responded to the item, "How successful was your attempt to get the person to agree with you?" $(1=$ not at all successful, to $9=$ completely successful).

Frequency. Participants responded to the item, "How often would you say that you use the tactic of saying the opposite of what you want to get others to agree with you?" Responses were given on a 9-point scale with each point on the scale labeled with a specific period of time (e.g., $1=$ never, $5=$ once per two months, $9=$ once per day).

\section{Results and discussion}

Of the 159 participants, 137 offered an example. Two independent raters evaluated whether these 137 examples constituted valid instances of SSA. For 105 cases, both raters agreed the example was valid. ${ }^{1}$ Overall rater agreement was $88 \%$, with 15 cases rated as not valid by both raters, and 17 cases where the raters disagreed. ${ }^{2}$ A sample of the examples identified by both raters as valid is offered below:

"When there is a choice between doing two things with a friend, I have told the other person to make the choice, hoping/knowing that they'd disagree and tell me I could choose (so I'd get my choice without looking too controlling)."

"One time I said that my friend had a good haircut when she didn't. Usually, she disagrees with my opinion so she changed it. Which was good."

"Before an important competition I have said before that I am extremely unprepared and unfit to compete at a high standard in the hope of hearing reassuring voices reminding me of how hard I had been working and how much I deserved it."

“Telling a partner (ex-girlfriend) that they didn't need me at all, to get her to say she did."

Among participants judged by both raters to have offered a valid example, the average difficulty of doing so was reported at $4.42(S D=2.30)$ on a 9-point scale where 9 represented no difficulty. These participants

\footnotetext{
${ }^{1}$ In six of the valid cases the participant provided an example that was a variant of the overweight/unattractive scenario that was illustrated in the questionnaire instructions.

${ }^{2}$ Because of the highly unequal number of valid and invalid cases in both studies, the kappa statistic is not appropriate. Thus rater agreement is expressed in percentages.
} 
reported the average success of the tactic at $6.60(S D=2.15)$ on a 9-point scale where 9 represented complete success. Participants reported their average frequency of using the SSA tactic at $5.41(S D=2.22)$. Given that a response of 5 was labeled as "once per two months" and 6 was labeled as "once per month," 5.41 on the frequency scale can be construed as use of the SSA tactic approximately 1.5 times per month. ${ }^{3}$

The results of Study 1 provide the first empirical evidence that people do use "reverse psychology" as a social influence tactic with some frequency in real-world influence settings. A majority of our participants $(105 / 159$, or $66 \%$ ) reported an example of SSA judged to be valid by two independent raters. Further, those participants who provided a valid example appeared to perceive SSA as a reasonably successful influence tactic, and reported using the tactic more than once per month on average.

As can be seen in the examples, however, the results of Study 1 suggest the existence of two seemingly distinct forms of SSA. The first form fits our initial conception - one who employs SSA against an influence target who is expected to contradict one's stated position in order to achieve generic attitudinal or behavioral compliance from the target (what we label hereafter as Persuasive Strategic Self-Anticonformity or PSSA). This form of SSA may essentially be a cold, rational choice taken in response to anticipated disagreement. The second form employs SSA primarily in hope of receiving reassuring or affirming messages from the influence target (what we label hereafter as Reassurance Strategic Self-Anticonformity or RSSA). Here, in essence, individuals appear to launch a "pre-emptive" attack on themselves, hoping that intimates will rush to defend them. RSSA may be particularly useful for attempts to gain reassurance because it is a relatively indirect form of influence. That is, by not going "on record" with a direct request for reassurance, the influence source is able to minimize threats to face needs should the desired reassurance not materialize (Brown \& Levinson, 1978).

To examine whether cases could be reliably categorized into these two types of SSA, the independent raters were instructed to nominate each case they declared valid as an example of either PSSA or RSSA. Of the 105 cases that both raters agreed were valid examples, 61 were nominated as PSSA by both raters and 36 were nominated as RSSA by both raters (with 8 cases of disagreement). Overall rater agreement was thus $92 \%$. Cases of SSA appear to be easily distinguished into persuasive and reassurance categories.

Although Study 1 suggests that the use of SSA is fairly prevalent, confidence in this conclusion is limited by the lack of a standard

\footnotetext{
${ }^{3}$ Difficulty of recall and frequency of use were positively correlated, $r=.23, p=.03$ (i.e., more frequent use was associated with easier recall). Success of the tactic was not significantly related to either difficulty of recall, $r=-.12$, or frequency of use, $r=-.01$.
} 
for comparison. In Study 2 we attempted to place the prevalence of SSA in context by comparing the use of this tactic to some of the social influence literature's better-established strategies. Participants were asked to provide examples of the use of the two forms of SSA identified in Study 1 (PSSA and RSSA), as well as examples of FITD, DITF, and DTR. We were able to provide cross-national evidence for the real-world use of PSSA and RSSA by collecting Study 2's data from Canada rather than Australia.

\section{STUDY 2}

\section{Method}

\section{Participants}

The sample consisted of 69 (52 female, 17 male) undergraduate students recruited from the University of Toronto participant pool who received course credit in exchange for participation.

\section{Procedure}

Participants arrived in groups, and following informed consent, completed the questionnaire package.

\section{Measures}

Social Influence Tactic Questionnaire. This questionnaire consisted of five sections. In each section participants were asked to recall an example of an occasion in which they had used one of the social influence tactics (i.e., PSSA, RSSA, FITD, DITF, and DTR). For each tactic, participants were given a description followed by a short example, then they were asked to provide an example of their own use of the tactic. Five versions of the questionnaire were used, each presenting the tactics in a different random order.

Difficulty. Participants were asked, "How difficult was it to come up with an example?" Responses were given on a 9-point scale where $1=$ not at all difficult and 9=extremely difficult (note this item was keyed in the opposite direction to Study 1).

Success. Participants responded to the item, "How successful was your attempt to get the person to agree with you?" Responses were given on a 9-point scale where $1=$ not at all successful and $9=$ completely successful. 
TABLE 1

Descriptive statistics for difficulty of recall, success of tactic, and frequency of use in Study 2

\begin{tabular}{lcccc}
\hline Tactic & $\begin{array}{c}\text { Percent valid } \\
\text { examples }\end{array}$ & $\begin{array}{c}\text { Difficulty } \\
\text { Mean }(S D)\end{array}$ & $\begin{array}{c}\text { Success } \\
\text { Mean }(S D)\end{array}$ & $\begin{array}{c}\text { Frequency }_{\text {Median }^{a} \text { (Range) }} \\
\text { PSSA }\end{array}$ \\
RSSA & $38 \%$ & $3.84(2.03)$ & $6.68(1.55)$ & $12(1-156)$ \\
FITD & $72 \%$ & $2.60(1.83)$ & $7.35(1.98)$ & $24(1-365)$ \\
DITF & $55 \%$ & $3.70(2.22)$ & $6.41(2.33)$ & $12(1-365)$ \\
DTR & $68 \%$ & $3.62(1.91)$ & $7.20(1.78)$ & $12(1-182)$ \\
\hline
\end{tabular}

${ }^{a}$ Medians are presented for frequency reports because of non-normal distributions of data.

Frequency. To better standardize reports of frequency of use, participants were asked, "How frequently would you say you use this tactic? (please give a concrete answer, such as 'twice per day' or 'once per year' and not something vague like 'often' or 'sometimes')." Participants gave openended responses, which were then converted into the average number of days per year each influence tactic is used. Thus higher scores on the frequency item equate to more frequent use of the tactic.

\section{Results and discussion}

Two independent raters judged the validity of the examples based on definitions provided by the researchers (see Table 1). For PSSA, both raters agreed the example was valid in 26 cases (with 6 cases mutually rated as not valid, 7 cases of disagreement, and 30 cases where no example was offered; rater agreement $=82 \%$ ). For RSSA, both raters judged the example as valid in 50 cases (with 2 cases mutually rated as not valid, 8 cases of disagreement, and 9 cases where no example was offered; rater agreement $=87 \%) .{ }^{4}$ For FITD, 38 cases were deemed valid by both raters (with 4 cases mutually rated as not valid, 5 cases of disagreement, and 22 cases where no example was offered; rater agreement $=89 \%$ ). For DITF, both raters agreed the example was valid in 47 cases (with 1 case mutually rated as not valid, 6 cases of disagreement, and 15 cases where no example was offered; rater agreement $=89 \%$ ). Finally, for DTR, the example was judged valid by both raters in 29 cases (with 8 cases mutually rated as not valid, 7 cases of disagreement, and 25 cases where no example was offered; rater

\footnotetext{
${ }^{4}$ In 10 cases the example of RSSA provided was a variant of the overweight/unattractive theme provided in the instructions. In addition, one example of DTR was also a variant of the illustration provided in the instructions.
} 
agreement $=84 \%$ ). Examples judged valid by both raters for each of the techniques are listed below:

PSSA

"I have used this tactic at work when I said I did not want to stay late. In order to do so I have said 'I want to stay late and get that time and a half and possibly double time' to convince the person that they are missing out on some good cash when in reality I did not want to stay and only said that so they would want the overtime."

RSSA

"I use this tactic after tests. I'll say 'I failed' so others will respond by saying something encouraging."

FITD

"I once used this tactic where I asked a friend if they could help me move my couch from one room to the next and after that asked if they could help me disassemble the door frame so the couch would fit and then move it in and reassemble the door frame."

\section{DITF}

"I've used this tactic before for things that require computer skills. I'll ask a friend to do something for me knowing they will not because it is time consuming. I follow my first request with a request to tell me how to do it so that I can stop bothering them and go do it myself."

\section{DTR}

"Once I forgot my bank card at home and was starving and out. So in order to get the money I explained to the person that 'the price of the food is that of one beer at a bar, you can sacrifice the price of one beer so I can eat' knowing the person was a heavy drinker and would probably drink 10 beers."

Descriptive statistics for difficulty of recall, success, and frequency of use for each tactic among participants judged by both raters to have reported a valid example can be seen in Table 1 . Correlation analyses among difficulty of recall, success, and frequency of use for all five tactics revealed only one significant correlation; a negative relation between difficulty of recall and frequency of use for DITF, $r=-.30, p=.05$. To compare the use of the subtypes of SSA to other tactics, paired-samples $t$-tests were conducted for participants who reported use of each pair of strategies. ${ }^{5}$ Among the participants who reported a valid example of both PSSA and RSSA, these tests revealed no significant difference for success $(d=0.26)$ or frequency

\footnotetext{
${ }^{5}$ Given the non-normal distribution of frequency data, results pertaining to frequency should be interpreted with caution.
} 
$(d=0.30)$ of the tactic, but RSSA examples were rated as easier to recall than PSSA examples, $t(20)=3.81, p<.01, d=0.84 .^{6}$

Among those reporting valid examples of each, PSSA examples were evaluated as significantly more difficult to recall than DITF, $t(19)=2.08$, $p=.05, d=0.48$. However, PSSA examples were evaluated as marginally easier to recall than DTR, $t(8)=2.09, p=.07, d=0.71$. No difference in recall difficulty was found between PSSA and FITD $(d=0.32)$. Further, PSSA did not differ on perceived success from FITD $(d=0.22)$, DITF $(d=0.11)$, or DTR $(d=0.24)$, nor did PSSA differ on frequency of use from FITD $(d=0.17)$, DITF $(d=0.07)$, or DTR $(d=0.09)$.

RSSA examples were rated as easier to recall than FITD, $t(27)=2.14$, $p=.04, d=0.42$, DITF, $t(36)=1.98, p=.06, d=0.33$, and DTR, $t(22)=3.44, p<.01, d=0.73$. RSSA was evaluated as more successful than FITD, $t(26)=2.87, p=.01, d=0.56$, but no differences in success were found with DITF $(d=0.08)$ or DTR $(d=0.18)$. Finally, RSSA was reported as marginally more frequently used than FITD, $t(27)=2.01, p=.06$, $d=0.41$, DITF, $t(34)=1.77, p=.09, d=0.31$, and DTR, $t(20)=2.04$, $p=.06, d=0.51$.

The results of Study 2 appear to confirm the real-world relevance of SSA. Participants found examples of the form of SSA used to seek interpersonal reassurance, RSSA, easier to recall than all other tactics studied and reported using RSSA more frequently than the three previously established influence tactics. Whereas valid examples of RSSA were provided by the largest percentage of participants $(72 \%)$, valid examples of PSSA were provided by the smallest percentage $(38 \%)$. However, this figure was roughly equivalent to the percentage of participants reporting DTR use $(42 \%)$, and suggests that PSSA does occur with some regularity in realworld influence settings. Indeed, self-reports of PSSA use among those who offered a valid example did not differ in frequency from the other previously established influence tactics.

\section{GENERAL DISCUSSION}

The results of our two studies suggest that SSA is an influence tactic that deserves closer study by social influence researchers. Sizable percentages of our participants were able to provide an example of their own use of SSA and were able to do so at rates equivalent with other influence tactics previously established in the literature. However, our work suggests that SSA is particularly likely to be used in the context of gaining

\footnotetext{
${ }^{6}$ Cohen's $d$ was computed using the calculator available at http://www.cognitiveflexibility.org/effectsize/ (Cepeda, 2008). This calculator accounts for Equation 8 from Morris and DeShon (2002) in order to correct for dependence between means.
} 
reassurance from others. The indirect nature of SSA may be particularly useful in sensitive interpersonal contexts as off-record requests can be used to protect individuals' face needs (Brown \& Levinson, 1978). In fact, the focus of social influence researchers on more direct influence strategies such as FITD and DITF may, to some extent, reflect a Western bias. In more interdependent cultures, where indirect communication and respect for face needs are especially highly valued (e.g., Wang \& Mallinckrodt, 2006), the ability to achieve one's goals through indirect communication may be especially important. Thus, data on SSA use from our Australian and Canadian samples may underestimate the prevalence of the use of this tactic in other parts of the world.

Although participants reported that both PSSA and RSSA were reasonably successful influence tactics, it is worth noting that both strategies carry inherent risks. With RSSA there is the possibility of your attempt backfiring; that is, the influence target may agree with you when you state the negative self-view you are hoping to have refuted. But even successful use of RSSA can have negative effects. Coyne (1976) argues that, among dysphoric individuals, solicitation of reassurance can lead to doubt in the validity of the positive feedback received because the reassurance seeker knows it was not provided with full autonomy. Over time, this can lead to an intensifying cycle of more need for reassurance, more doubt over that reassurance, and ultimately frustration, helplessness, and depression (Shaver, Schachner, \& Mikulincer, 2005). Thus RSSA seems like a tactic that might best be used sparingly.

PSSA also has the potential to backfire if the target of influence agrees with your initial, disingenuous request. Research suggests that individuals are reasonably successful at tacit coordination, or anticipating others' behavior and adjusting their own actions accordingly (Wittenbaum, Stasser, \& Merry, 1996). Thus such backfires may be more rare than assumed. However, once again, even successful PSSA attempts can have negative consequences such as the imposition of unequal power dynamics (through withholding of information) or feelings of inauthenticity. Thus, although PSSA appears to be one way to successfully gain influence over a resistant target, there are other methods one could use that allow for higher degrees of directness. For example, Knowles and Riner (2007) provide evidence that simply acknowledging another's feelings of resistance can increase the likelihood of successful influence in such a situation.

Of course, the present research is rather preliminary, and not surprisingly contains limitations. First, our measures of difficulty of recall, success of a tactic, and frequency of use are all single items, which calls into question their reliability. Second, our reports of the use of SSA and the other influence tactics rely exclusively on self-report, retrospective accounts. If some tactics are executed without full conscious awareness or are not fully 
encoded into memory, these tactics may be under-reported here. For example, to the extent that PSSA involves a degree of manipulation that is inconsistent with one's self-image, this tactic may not be fully acknowledged at the time of influence or may not be highly accessible in memory at the time of recall. Third, it is important to note that our samples consisted exclusively of undergraduates, and thus it is unclear whether the present results would generalize to broader populations. Of course, there may well be some groups for whom SSA is used more frequently than in our student sample (e.g., parents dealing with children in the "terrible-twos,": more formally, Erikson's, 1963, second psychosocial stage, autonomy vs shame and doubt). Finally, there is nothing in our data set that can inform us, objectively, as to the prospects of successful influence using SSA.

Whereas our studies suggest that SSA is a relevant, real-world influence tactic, further research will be needed to expand on the dynamics of this form of influence. For example, one hypothesis suggested in the introduction is that individuals who are perceived as disagreeable will be especially likely to be targeted with PSSA tactics. On the other hand, SSA used with the goal of receiving reassurance may be especially likely to be targeted at highly agreeable individuals. It would also be valuable to conduct future research using observational or experimental methods. For example, participants could be asked to influence a research confederate who is either consistently agreeable or disagreeable. Over the course of multiple influence attempts, we would predict that participants might begin to make PSSA attempts towards the disagreeable confederate. Such a result would confirm our notion of the $\mathrm{S}-\mathrm{R}$ link between anticonformity as a stimulus and self-anticonformity as a response.

Overall, the current studies suggest that SSA merits further research attention. Our data show that people do appear to consciously misrepresent themselves in order to influence others. More generally, the existence of SSA reaffirms the notion that social influence researchers need to attend to the fact that in real-world social influence situations, people will spontaneously present public positions that differ from their privately held views (MacDonald \& Nail, 2005). In the case of SSA, this misrepresentation may be a very conscious choice of influence strategy.

Manuscript received 26 April 2010 Manuscript accepted 17 August 2010 First published online 12 October 2010

\section{REFERENCES}

Ascher, L. M. (1989). Therapeutic paradox. New York: Guilford Press.

Brehm, J. W. (1966). A theory of psychological reactance. New York: Academic Press. 
Brown, P., \& Levinson, S. C. (1978). Politeness: Some universals in language. New York: Cambridge University Press.

Cepeda, N. J. (2008, June). Effect size calculator [computer software]. Retrieved July 1, 2010 from http://cognitiveflexibility.org/effectsize

Cialdini, R. B., Vincent, J. E., Lewis, S. K., Catala, J., Wheeler, D., \& Darby, B. L. (1975). Reciprocal concessions procedure for inducing compliance: The door-inthe-face technique. Journal of Personality and Social Psychology, 31, 206-215.

Coyne, J. C. (1976). Toward an interactional description of depression. Psychiatry: Journal for the Study of Interpersonal Processes, 39, 28-40.

Davis, B. P., \& Knowles, E. S. (1999). A disrupt-then-reframe technique of social influence. Journal of Personality and Social Psychology, 76, 192-199.

Erikson, E. H. (1963). Childhood and society. New York: Norton.

Frankl, V. E. (1967). Logotherapy. Israel Annals of Psychiatry and Related Disciplines, 5, 142-155.

Freedman, J. L., \& Fraser, S. C. (1966). Compliance without pressure: The foot-inthe-door technique. Journal of Personality and Social Psychology, 4, 195-203.

Knowles, E. S., \& Riner, D. D. (2007). Omega approaches to persuasion: Overcoming resistance. In A. R. Pratkanis (Ed.), The science of social influence: Advances and future progress (pp. 83-114). Philadelphia, PA: Psychology Press.

Loriedo, C., \& Vella, G. (1992). Paradox and the family system. New York: Brunner/ Mazel.

MacDonald, G., \& Nail, P. R. (2005). Attitude change and the public-private attitude distinction. British Journal of Social Psychology, 44, 15-28.

Morris, S. B., \& DeShon, R. P. (2002). Combining effect size estimates in metaanalysis with repeated measures and independent-groups designs. Psychological Methods, 7, 105-125.

Nail, P. R., MacDonald, G., \& Levy, D. A. (2000). Proposal of a four-dimensional model of social response. Psychological Bulletin, 126, 454-470.

Nail, P. R., \& Van Leeuwen, M. D. (1993). An analysis and restructuring of the diamond model of social response. Personality and Social Psychology Bulletin, $19,106-116$.

Shaver, P. R., Schachner, D. A., \& Mikulincer, M. (2005). Attachment style, excessive reassurance seeking, relationship processes, and depression. Personality and Social Psychology Bulletin, 31, 343-359.

Tillis, M., \& Wager, W. (1984). Stutterin' boy: The autobiography of Mel Tillis. New York: Rawson Associates.

Wang, C. C. D. C., \& Mallinckrodt, B. S. (2006). Differences between Taiwanese and U.S. cultural beliefs about ideal adult attachment. Journal of Counseling Psychology, 53, 192-204.

Willis, R. H. (1965). Conformity, independence, and anticonformity. Human Relations, 18, 373-388.

Wittenbaum, G. M., Stasser, G., \& Merry, C. J. (1996). Tacit coordination in anticipation of small group task completion. Journal of Experimental Social Psychology, 32, 129-152. 
Copyright of Social Influence is the property of Psychology Press (UK) and its content may not be copied or emailed to multiple sites or posted to a listserv without the copyright holder's express written permission. However, users may print, download, or email articles for individual use. 